\title{
La Crónica y los Estudios Culturales. Notas para un debate compartido
}

\author{
Dr. Tanius Karam ${ }^{1}$ \\ Academia de Comunicación y Cultura/ \\ Universidad Autónoma de la Ciudad de México \\ tanius@yahoo.com
}

\begin{abstract}
Resumen: En este trabajo se reflexiona sobre los modos de articulación entre los estudios culturales y la crónica, para ello se hace una recapitulación de lo que este movimiento ha significado, algunos de sus rasgos y de manera especial su importancia para el campo académico de la comunicación. En la segunda parte se describe de la misma forma a la crónica y algunos rasgos para su definición. Finalmente se consideran en forma de lista algunos núcleos para un debate sobre los modos de articulación que implican el sentido del lenguaje científico y los modos de relación entre sociología, comunicación y literatura
\end{abstract}

Palabras Claves: Estudios Culturales, Crónica, Discurso, Crítica, Comunicación.

Abstract: In this word we reflect about ways of link cultural studies and chronicle, for this we make a synthesis about this movement meant, as well some of its features, mainly how social communication academic field have been affected. At second part, we describe as same way, chronicle and some of its feature. Thirdly we consider some problematic field, we are trying to address a debate about ways of link our main two concepts, which implies jeans for defining scientific language and interdisciplinary discussion among sociology, communication and literature studies

Key Words: Cultural Studies, Chronicle, Discourse Critics, Communication.

\footnotetext{
${ }^{1}$ Doctor en Ciencias de la Información por la Universidad Complutense, Madrid. Profesor e investigador en la Academia de Comunicación y Cultura en la Universidad Autónoma de la Ciudad de México y del Centro de Estudios sobre la Ciudad en la misma universidad. Colabora en varias suplementos y revistas, como la Sección de Cultura" de la Revista Siempre (ciudad de México), artículos sobre literatura y periodismo mexicano. Colaborador en las Revistas Vida Nueva de Madrid, artículos, reportajes y entrevistas en torno a Religión, Cultura y Sociedad en México.
} 
Sueles con frecuencia pensarse el fenómeno de los estudios culturales (EC) como una escuela u orientación, por lo general de tradición anglosajona, que ha impactado a las humanidades y las ciencias sociales en diversas formas. De una primera fascinación y gran divulgación cada vez con más frecuencia se alzan voces muy críticas contra esta tendencia por lo que por ejemplo se les puede acusar de una "razón perezosa” ( $C f$. Sousa Santos). Los EC tienen un rango de preocupaciones muy extensos, en el que prácticamente cualquier problema social y cultural puede quedar incluido, además se imponen nuevas formas discursivas que se consideran más literarios que estrictamente sociológicas o antropológicas, de la misma forma se cuestiona la distancia entre la producción científica y la realizada por actores sociales sin este bagaje lo que lleva a discutir el tema del poder y el conocimiento como un aspecto fundamental en la producción de la información y el conocimiento

En lo que parece existe un consenso es lo indiscutible es la influencia e importancia, de manera especial, para los estudios en comunicación, los cuales prácticamente se han reorganizado a partir de su encuentro con los EC. En medio de estos hechos, la crónica, en tanto objeto y dispositivo discursivo cumple un papel importante lo mismo para criticar o ponderar a los EC. En este dilema más que una postura específica queremos señalar, primero para las categorías básicas de EC y par el estudio de la crónica algunos núcleos problemáticos, para luego esbozar dentro de un eje de debates que lejos de desarrollar enlistamos bajo algunos argumentos.

\section{Recapitulaciones sobre los Estudios Culturales}

Si bien existe un razonable grado de consenso entre estudiosos de la cultura que los EC provienen de la vertiente anglosajona, impulsada (no creada del todo) desde el celebérrimo Centro para el Estudios de las Culturas Contemporáneas (CECC), luego su arribo a los EE.UU. (en los setenta) y su institucionalización (aparición de departamentos, revistas especializadas, etc.) lo que va facilitar su exportación a otras partes del mundo, en especial a América Latina, donde por cierto (hay que subrayarlo) no llega el proyecto crítico original de los EC desde sus fuentes, sino sus mediaciones y relecturas.

En su origen, esto que se llamó EC, se erigió como una "cuasi-disciplina" e hizo explosión con sus programas y asociaciones; en realidad no siempre se 
reflexiona la forma en que este movimiento que colisionó con otros, los afectó o bien posibilitó otras corrientes. Bien podemos decir que los EC son un signo de una serie de fenómenos, un “condensador”, entendido más que como síntesis y encuentro dinámico de varias formas de comprender la realidad y discursar sobre ella y desde los EC se expanden hacia otros aspectos, que como ha sucedido no siempre guardan relación con la fuerza originaria y en el camino se resemantizan o adaptan a realidades y necesidades locales. ¿Qué logran los EC como agrupación y expansión, por qué su éxito y difusión, su gran acogida y para el caso, por ejemplo, del campo académico de la comunicación, su papel central en lo que consideramos un nuevo acicate dentro de esta área de estudios y una refundación de sus estudios?

Con relación a esta última pregunta, Galindo (2003) señala para los estudios de comunicación que tras el estudio de los efectos socio-políticos que fueran tan importante para la Mass Communication Research y sus hitos conformadores en Klapper o Lazarsfeld, dan paso a otro hito en los EC. Esta corriente va mostrar un interés por casi cualquier asunto relacionado con la vida (principalmente urbana) de la segunda parte del siglo XX les interesa. Aunque surgen con énfasis local, su vocación es de generalización sobre las condiciones de construcción de la vida social y simbólica de los actores que comparten los fenómenos de lo nacional, transnacionales. No es fácil centrar a los EC en temáticas dominantes, pero pueden ser nombrados desde sus intenciones críticas y emancipadores; los EC son más propiamente un archipiélago, de esta manera tocan a la comunicación en principio por el estudio de sus públicos y los fenómenos de configuración socio-cultural por el contacto e impacto de los medios y en el camino han privilegiado cuatro objetos de estudio (jóvenes, género, minorías y movimientos sociales). Su perspectiva abarcadora alcanza aspectos históricos, estéticos, ecológicos; no hay que olvidar que todo comenzó en un departamento de estudios literarios, por tanto si una caracterización más precisa fuera posible, habría que subrayar este acento estético y literario en su origen.

Los EC fueron el signo que hizo emerger nuevos temas en el debate y en las áreas de producción de saber, caracterizado por la superación de las disciplinas "ego"-céntricas (las que responden únicamente a los rasgos de una ciencia "moderna”, “europea” y "masculina”). Con los EC se dimensionó la importancia del 
análisis histórico local, asociado con una nueva actitud hermenéutica y la estimación de los valores asociados con las realizaciones tecnológicas y su relación con otros valores. En la formulación del problema de las "dos culturas”2 estaba el supuesto implícito de que la "Ciencia" era más racional, "más dura", más precisa, más poderosa, más seria, más eficaz y de mayores implicaciones que la filosofía, las artes o las letras (hubo por cierto disciplinas que se adscribieron a esta visión como los estudios literarios, la antropología y personas dedicadas a los pueblos olvidados por la "modernidad"). La premisa latente era que la ciencia era más moderna, más europea y más masculina; contra estas visiones reaccionaron los estudiosos de género y todos los estudios no eurocéntricos al proponer nuevas visiones y demandas en la revaloración de los EC.

Con la proliferación de los EC sobrevino la crítica a los academicismos, al locus de la práctica intelectual con tendencia más complaciente que crítica al poder y sus distintas formas. Así los EC activaron la diversidad de articulaciones heterogéneas mediante una práctica intelectual que abogó por desbordar el refugio academicista para intervenir en los conflictos de valores, significaciones y poder para hacer un enfoque crítico latinoamericano que superara cualquier reduccionismo teórico, las fáciles polarizaciones o la sobre-dimensión de algunos categorías. Lo crítico y lo cultural se erigieron como en un esfuerzo por clausurar los saberes corporativos para poner a circular sus desacuerdos con el presente por redes amplias de intervención en el debate público, pero también una crítica vigilante de sus lenguajes que no quiere mimetizarse con la superficialidad mediática de la actualidad. Así las voces de la resistencia y la oposición se ven con otra perspectiva, como un conglomerado de tensiones (entre el proyecto académico de los estudios culturales y la crítica política de la cultura) de la cual aparece una nueva riqueza que permite potenciar tanto el estudio como la lucha misma de ciertos espacios (Richard citado por Ochoa, 2002, p. 4).

En suma, que sobre el apunte de los EC como saber o cuasi-disciplina, son un fuerte signo de una serie de transformaciones. Criticados como "razón perezosa",

\footnotetext{
${ }^{2}$ C.P.Snow que quiere decir dos modos de conocimientos, uno "positivo" y otro especulativo; no "científico" y otro que no lo es (puede ser teológico, intuitivo...) (Wallerstein, 1996: 5). Esto se vincula con una idea rígida de lo que es y no es la ciencia a partir del s.XVII, de los objetivos legítimos del supuesto saber científica (ciencia, razón, progreso...)
} 
o bien como simple literatura, es algo que no a muchos - ubicados canónicamente en esta corriente- no interesa o preocupa. El "malestar" de los EC que apunta García Canclini, parece ser más exógeno que interno, porque también es cierto que al interior de quienes se asumen en este paradigma privan un conjunto de preocupaciones muy distintas a las que parecen llamar la atención a críticos y detractores, además es algo previsto o considerado por ellos.

\section{Rasgos sobre la crónica como dispositivo múltiple: presencia, trasgresión, fractura, fugacidad...}

Se acepta en una primera mirada a la crónica como un género informativo porque funciona básicamente con hechos, a diferencia de la crítica literaria o el artículo que funciona con juicios y argumentos. De las definiciones más funcionales en los manuales periodísticos mexicanos tenemos la división que Leñero y Marín (1986: 156) resume que la crónica puede ser "informativa", "opinativa" o "interpretativa" vistos como tres significados distintos donde el "entorno" juega alternativamente la dimensión fáctica, valorativa y experiencia del narrador y lo que le rodea, y al mismo tiempo se articulan niveles distintos en la comprensión y explicación de la realidad. De manera muy poco crítica, las crónicas pueden subdividirse de acuerdo a otros criterios más funcionales como el tema central (se habla así de crónica deportiva, política, social, taurina...), por su contenido (noticiosa, parlamentaria, deportiva, sociales, local, nota roja, crónica cultural, literaria) o por su estructura (deductiva, inductiva...). Pero una primera revisión detallada a estas nociones muestra problemas y severas imprecisiones, sobre todo en la evolución más reciente del género lo mismo en la historia que en la literatura, en la antropología que en el periodismo, y es básicamente los modos interdiscursivos de sus usos donde radica una parte del problema y que vale para algunos como crítica. Por ejemplo el crítico literario Domínguez Michael (citado por Egan, 2001: 81) observa que el uso y abuso de la forma ha redundado en su dificultad para definirla; abuso que no es para nada reciente y data del siglo XIX. Los usos pragmáticos de la crónica siempre han sido diversos: de escaparate a púlpito, de telenovela privada a ejercicio de observación participante; de cualquier forma, esto refleja lo que ha sido la misma historia de la crónica y que confirme para odios y beneficios de muchos el potencial del género. Para uno de los cronistas mexicanos más emblemático, Carlos Monsiváis, 
uno de los logros de la crónica reciente es el uso periodístico abundante para dar cuenta de actores, movimientos sociales y luchas de las minorías ( $C f$. Aranda, 1991: 45-46), que han hecho de la crónica un instrumento útil para el conocimiento de la realidad social.

La crónica es uno de los géneros más importantes en la historia de la literatura y el periodismo. Inserto en esa frontera que puede molestar a los amantes de las categorizaciones, el género se caracteriza por su mestizaje y sus posibilidades expresivas. Parafraseando aquella imagen que Paz (1993) hacía de la "llama doble" (la llama del amor y del erotismo) podemos usar esa figura del Nóbel para describir esa doble flama que forma no una, sino varias llamas dobles: ficción y realidad; oralidad y literalidad; presente y pasado; literatura y periodismo; empírico y poético. Esta personalidad difícil de sostener ha hecho que la crítica la arroje hacia un limbo en el cual su condena o su aceptación no acaban por quedar claro. Parte de la confusión puede deberse a la vecindad que establece con el ensayo, la noticia, el testimonio y el cuento, lo que ha creado la variedad de nombres que se usan en su sinonimia como ficción documental, cronovela, meta-periodismo, relato no ficcional, periodismo interpretativo, neocostumbrismo, no [crónica]vela, literatura testimonial, etc. (Cf. Egan, 2001: 80).

(a) Si nos atenemos a la definición etimológica del término, crónica remite a lo temporal. Reguillo (2003: 61) propone una distinción entre la crónica y lo crónico. La primera acepción remite a la relación ordenada de los hechos; y en masculino, lo crónica, como enfermedad larga y duradera. Este primer elemento sugiere lo equívoco del término o su dificultad para dejarse asir por una concepción restringida. En este sentido lo temporal puede remitir a lo inmediato o lo permanente. Monsiváis (1980) gusta definir a la crónica por un verso de Quevedo, "sólo lo fugitivo permanece". Lo crónico remite ciertamente a una serie de acontecimientos más o menos pautados, de los cuales se da cuenta sobre, más que de lo específico o lo contextual, de su tensión dinámica. Estas dos nociones de temporalidad se anudan dialécticamente en lo que con frecuencia se revela como fuerza o sanción, como evidencia e ironía.

(b) Dentro de las múltiples tensiones que podemos encontrar en la crónica, una de ellas se ancla en el eje oral-escrito. La crónica se asocia con frecuencia a lo 
oral, al relato vivencial, a la experiencia que sólo se puede conocer por voz de quien lo vivió (por lo general para el caso del enorme cuerpo de textos en la literatura testimonial en América Latina, suelen ser hechos trágicos y violentos).

Por otra parte, a nivel más amplio de varios géneros mediáticos, asistimos a un resurgimiento de lo testimonial, del valor depositado en la palabra de quien vivió los hechos como una referencia inmediata a la certeza periodística y al mecanismo de certidumbre a través de otro o un sí mismo reconfigurado para el relato. Wieviorka (citada por Salazar, 2003) resume esta tendencia de los testimonial como una "era del testigo" la cual sobreviene como una necesidad ética de construir una cultura crítica que impida, por el ejemplo, para el caso de acontecimientos violentos y/o aterradores, que éstos se repitan como el dramático caso de las dictaduras latinoamericanas que han generado una especie de estallido de la memoria, al inventario de los detalles privados de un recuerdo que denuncia y tiene importancia pública.

La literatura testimonial va ser uno de los aspectos que más se impulsan hoy día la literatura latinoamericana. El yo ultrajado que revive los hechos violación a los derechos humanos y ese relato por sí mismo, supera cualquier posibilidad de la ficción, deja muda la creación o se conforma una "meta-creación" en el sentido de una reconstrucción donde el dato y el detalle aglutina más que la descripción. El testimonio confiere un valor al informante dentro del contrato de la escritura; el rol de escritor cambia y su labor será más efectiva cuanto dé cuenta con mayor grado de variedad y matices los intersticios del recuerdo horrendo. Pero este recuerdo y testimonio interpela de forma aguda la promesa del proyecto iluminista, cuestiona la respuesta única a la representación unívoca del mundo; reorganiza la manera de entender el mundo y deja ver el dolor de actores ignorados y poco atendidos por las instituciones dominantes.

(b) Vinculado con la idea de temporalidad, podemos destacar los modos para registrar la velocidad, la simultaneidad de los tiempos, como otra posibilidad de la crónica para dar cuenta lo mismo de la permanente que de lo fugaz, más aún, mostrar estos movimientos como en una tensión dinámica. Décadas atrás, Ortega y Gasset en La rebelión de las masas advierte cómo esta rapidez lleva a intercambios más dinámicos en los distintos niveles de la realidad, llama la atención sobre el 
ascenso histórico y los nuevos movimientos culturales de un consumo masificado y estandarizado. Una estrategia para soportar estos planos y desniveles será necesaria una mirada "multi" o "pluri" disciplinaria, pero no como una acción deliberada sino como parte de un proceso en el que al narrar el lenguaje y el espacio, la acción y la expresión, eso necesariamente será parte, a la hora del análisis, de un acercamiento múltiple y complejo.

(c) El fenómeno anteriormente señalado no sería posible sin una cierta condición de "flexibilidad", la cual históricamente ha mostrado su capacidad para plegarse y ha sabido sobrevivir a mutaciones y cambios. A diferencia de la ponderación del saber vehiculado por los géneros de información o el puro comentario modelado desde los "géneros opinativos"; más que "hibrides", la crónica es "plasticidad" ante los sistemas de representación dominante, antes las formas convencionales en la articulación de lo público o lo privado, de lo macro y lo micro. La crónica opta por la intensidad, pero sabe jugar con la extensión cuando más que detalle, se regodea en los contextos y las atmósferas. Se asume el punto de vista personal como el único, el cual se presenta más vinculado a la experiencia que al saber, a la presencia y al testimonio que a la "verdad". A nivel de redacción, la primera persona o los estilos libres han mostrado ser más recurrentes y desde ahí la construcción de un "yo" más esquivo. Para Salazar (2005), la crónica es contingencia y parcialidad que se realiza a través de una enunciación que transgrede la objetividad clásica.

(d) Reguillo (2003) opta por una caracterización doble de la crónica, doble movimiento de encuentro y disrupción. Con la crónica aparece un nuevo tipo de discurso transversal no reducible a las célebre dicotomías para caracterizar los modos enunciativos del discurso periodístico o literario (ficcional / no ficcional; oral / escrito; información / opinión...) donde radica parte de su riqueza y efecto discursivo único. Esta nueva forma que parece visibilizarse desde la crónica como un nuevo espacio público. De su capacidad para hacerse cargo de las transformaciones en las formas del relato, en las sensibilidades, en las formas de comunicar de los otros, depende en buena medida que lo proscrito o lo estigmatizado, lo invisibilizado o lo otro, emerja con fuerza para abrir la posibilidad de re-pensar un proyecto 
modernizador que afirmó sus dominios mediante la condena al silencio de amplios sectores de la sociedad.

Si bien algunos discursos más tradicionales y convencionales buscan reducir la complejidad del mundo sometiendo los lenguajes irruptivos a una tipificación normalizada, la crónica por el contrario, buscaría abrirse a esa complejidad, de ahí el recelo del que es objeto, las sospechas que sobrevienen a su uso en las rutinas periodísticas, académicas, literarias, cuya operación discursiva principal ha sido la de domesticar lo "desconocido" mediante el sometimiento referencial a marcos cercanos y conocidos. Lejos de la sospecha, Reguillo postula a la crónica como género integrador, definido y delimitado que se caracteriza convocar distintos modos del lenguaje, como espacio de evocación compartida, y vehículo de socialización que tiende puentes entre mundos diversos.

(e) Con frecuencia el primer rasgo que se señala de la crónica es su lugar de origen. Hemos querido dejar este aspecto en nuestro último apartado, para poder comprender los aspectos que describen su origen con los rasgos que hemos señalado en los cuatro puntos anteriores, que más que aspectos, son problemas, categorías descriptivas de un género visto como proceso. De forma convencional se acepta a la crónica como un género seminal en la historia de la literatura hispanoamericana, uno de los primeros dispositivos que surgen en el nuevo mundo. Si bien esto no es del todo falso, para Rotker (2005) es impreciso ubicar su origen en los Cronistas de Indias; como el caso del estudio científico de la comunicación (toda proporción guardada), si bien su origen es muy anterior a su institucionalización, su difusión es reciente; de la misma manera con la crónica, la cual subsiste desde hace varios siglos en formas que no siempre son las canónicas para referirse y postular al género, ni tampoco en usos y estilos que hoy hemos definido como centrales. También es cierto que todo modo de definición en historia, literatura o periodismo implica una interpretación selectiva y una manera de colocarse delante de la práctica discursiva que porta; así ver a la crónica en América Latina ya en los textos del siglo XVI es una manera de interpretarla, a diferencia de lo que Rotker (2005) propone buscando su origen no en el siglo XVI sino a finales del XIX, en el contexto no de la contrarreforma sino del modernismo. 
En su ensayo, dedicado en principio a la crónica, dedica varios capítulos, a describir los rasgos y modos de este modernismo latinoamericano, en la importancia que tuvo e implicó, en las relaciones que guardar con los procesos de industrialización y urbanización que vivían varios países de la región. Estos procesos obedecen entre otros a la integración de las Españas en el mundo burgués, la lenta disolución de la sociedad tradicional y la paulatina formación de la sociedad burguesa; también a la obsesión posindependentista y romántica por la originalidad sumada al deseo por adquirir el derecho a cualquier escenario del universo y a la individualidad, sin por eso dejar los problemas de independencia y representantividad (Rama citado por Rotker, 2005: 23). Estas definiciones arraigadas en la historia de las ideas intelectuales, propician una lectura que integre la práctica social al lenguaje, las necesidades de reproducción y la prensa, los nuevos vínculos entre texto y contexto.

Rotker sugiere que la crónica es sustancial a estas transformaciones que se condensan en el modernismo. Parece interesante ver como muchos de los rasgos aceptados por la crítica para definir al modernismo (Búsqueda de los menos convencional, renovación permanente, audacias temáticas, registro de matices, mezcla de sensaciones) no parecen disonantes a lo que hemos dicho sobre la crónica hasta al momento. Otro argumento a favor de esta estrecha relación entre modernismo y crónica es que prácticamente todos los poetas, (siempre con algunas excepciones) por hablar de la forma que entonces se considerada como más desarrollada dentro de la literatura de la época, ejercieron el periodismo y se valieron de la crónica (por citar algunos de los casos más emblemáticos: Gutiérrez Najera, Nervo, Del Casal, Luis. G Urbina, J.J. Tablada, J.E. Rodó) como dispositivo variado y forma para combinar los gusto formales con las urgencias o necesidades referenciales.

\section{La crónica y las formas de relaciones con los Estudios Culturales}

Optamos para explorar las relaciones explícitas y latentes entre la crónica y los estudios culturales un listado un tanto azaroso de núcleos problemáticos de estos intersticios y planos, de un debate que trasciende los ámbitos teóricos para internarse 
también en las formas de enunciación de lo que hoy se entiende por disciplina y donde muchos tipos de escritura se cuestionan unas a otras.

(a) Con frecuencia de olvida el origen literario de los llamados Estudios Culturales y lo que fue el Centro de Estudios para las Culturas contemporáneo de Birmingham. Williams y Hoggart venían de los estudios literarios y fue Hall, quien internacionaliza el Centro, saca a los investigadores a la calle, y genera un diálogo y debates que hoy son centrales para los estudios en comunicación. Una de las obras señeras de estos founding fathers. Hoggart estudia en su clásico The Uses of Literacy la influencia de la cultura difundida entre la clase obrera y los medios de comunicación: analiza cómo las publicaciones destinadas a este público, se integran en este contexto. Es decir, crítica la subestimación que se hace de los productos consumidos por estos grupos y postula que hay procesos al interior que no son tan evidentes como los que a veces de forma prejuiciosa se hace. De la misma forma, el nuevo concepto que vehicula Williams en el otro clásico (Culture and Society) postula una definición de la cultura en la sociedad industrial; al explorar conceptos como "cultura", "masas", "arte" asiente la historia de las ideas sobre una historia del trabajo social de producción ideológica. Las nociones, las prácticas y las formas culturales materializan visiones y actitudes que expresan regímenes, sistemas de percepción y sensibilidad. Esta es la idea central que va desarrollar en The Long Revolution donde subraya el papel de los medios de comunicación dentro de la dinámica del cambio social.

Estudios Culturales y la crónica surgen de ámbitos de producción y de difusión que parecen distintos, pero como hemos observado presentan aspectos similares. No resulta causal que cronistas con facilidad se les reconozcas “culturalistas" (claro, sin ellos asumirse como tal); a la inversa, que los EC vean en la crónica (literaria, periodística, histórico...) un dispositivo de análisis idóneo para el estudio de las identidades culturales, una fuente legítima para la investigación central en las conclusiones. Esta flexibilidad de la crónica es mutatis mutandi (toda proporción guardada) parecida a la que (para bien o para mal) tienen los estudios culturales, de aquí su impacto y la facilidad de su difusión.

Para la crónica, de impronta más administrativa en el s. XVI, pasa (casi simultáneamente) a ser relato de viaje, para luego confundirse con el periodismo y la 
lucha política en el siglo XIX y más de medio siglo después, hoy claramente asociada con la literatura testimonial o como vehículo de movimientos sociales. Los EC surgen como una crítica al establishment, dentro del movimiento de la nueva izquierda para igualmente confundirse como otros saberes y objetos; comienzan con un proyecto crítico que lo vincula a la nueva izquierda, pero al institucionalizarse y diseminarse se producen relecturas, muchas de ellas alejadas.

En estos juegos de idas y vueltas, similitudes y semejanzas, de orígenes compartidos, la literatura (y sus variantes semántica) en tanto como fenómeno social central en la definición de las identidades culturales es una variable conflictiva y problemática. Lo literario (entendido por ahora de una forma muy amplia: como estética y fenómeno socio-cultural) es posibilidad de abrirse a lo no dicho por otros saberes, al grado que no pocos una forma de mirar a los objetos que se traduce en un compromiso el cual que no es únicamente académico o periodístico. Como en el siglo XIX, la literatura se convierte en un espacio central para advertir y dar cuenta de procesos que apenas el pensamiento social se atreve a nombrar; dentro de este siglo, el periodismo (y cabe señalar que sin duda el "siglo de oro" de esta actividad pública parece que será el XIX) es un mosaico diverso para dar cuenta de temporalidades y hábitos, señales y consignas, donde se mueven las necesidad del espacio público, se aglutinan grupos y se dimensionan programas e ideas.

(b) Los EC analizan las formas explícitas o difusas de persistencia cultural, se apoyan para ello en investigaciones orales y las culturales locales, las cuales se estudiante en su resistencia u oposición a las formas legitimadas a la "cultura sin rostro" (para usar la expresión de Hoggart para hablar de cultura de masas). Estos temas de discusión se han comprometido con perspectivas de reconstrucción de los discursos de dominantes y desde los EC se reconstruirían las historias de procesos socio-político y confrontaciones de clase. Para los EC tiene especial importancia temas como ideología y lenguaje, lo simbólico y el poder, lo cual es indisoluble de sus formas expresivas. El problema parece ser que no se establecen diferencias ente conjuntos textuales producidos en un entorno y en otro, ambos son válidos, legítimos; más aún, cuanto más heterodoxo y "crítico" (entendido esto como antipoder o anti-orden establecido), parece en sí mismo un componente positivo. Lo que hay en el fondo de este debate es la problematización sobre las formas de articular 
teoría-práctica, acción social-acción científica-acción política; esos aspectos más que formales, sustanciales para los estudios culturales a los que muchos exponentes han quizá renunciado, es lo que al ubicar es posible hallar una relación más madura y menos folklórica o "políticamente correcta" para relacionar a la crónica con los estudios culturales para quienes los constructos "diversidad", "diferencia" son centrales que permiten un nuevo logos en la escritura, las cual puede traducirse (como ha sido en algunos casos) con una sintaxis y forma novedosa, confrontante y singular, y que por tanto dichas narrativas resulten incómodas para las instituciones y fuerzas dominantes en el campo cultural.

(c) La crónica puede ser un instrumento sugerente para hacer un análisis "comunicológico" en el sentido de interacción (como un objeto posible dentro de la ciencia de la comunicación). Con la producción de estos textos se generan redes de enunciación: por ejemplo, el caso de Francisco López de Gómara (Historia general de la Indias y Conquista de México) y Bernal Díaz del Castillo; si el primero no hubiera escrito su crónica quizá Bernal se hubiera conformado con describir oralmente su historia. Más que un "contar" a Bernal le interesa un "desmentir”. A un nivel más complejo se puede ver la "interacción" dialógica de los textos producidos. Esto se realiza cuando se analiza comparativamente la construcción / interpretación de las diferencias y desigualdades socioculturales lo mismo en los Cronistas de Indias que en los textos de los grupos originarios. Con cierto maniqueísmo Todorov (1999) ha aproximada ya un acercamiento en este sentido. Le ha faltado sobre la parte de las crónicas de los amerindios, en donde a diferencia de los conquistadores, sobre sale un sentido expresivo y emotivo mucho más complejo. De la misma forma cada conquistador, misionero o cronista genera sistemas de fuerzas cruzados donde hallamos formas de autorrepresentación y heterorrepresentación muy diferenciados. En algunos casos, como los cronistas de indias, la descripción supuso compromisos con los pueblos conquistados (F. Bernardino de Sahagun) y denunció injusticia, en otros (como en las crónicas escritas por F. Diego de Landa) hay esfuerzos denodados por ajustar y matices, por hacer comunicables. De estos intentos convergentes, uno de los más significativos acaso es el del Inca Gracilazo de la Vega quien tiene en su obra principios para comprender la comunicación intercultural de nuestros días. 
Este acercamiento que asomamos es un modo, que desde la crónica se abre para el historia de procesos sociales desde una dimensión interactiva y a fin de cuentas en la evolución del propio pensamiento. Valenzuela sugiere cómo la crónica es central en este proceso de reconocerse y aprender a ver al otro. El cuerpo de textos derivados como el principio de una historia que si bien es escrita por los dominados aun para entender ésta no se puede desentender de la voz dominada y viceversa.

(c) Valenzuela (2003) encuentra otro modo de vincular EC y crónica a través del problema de la multiculturalidad. Los EC subrayan la interpretación de las transformaciones en la producción histórico-social de los discursos. Ahora bien, como tantos aspectos en la terminología de los estudios culturales hay que hacer diferencias y matices. La multiculturalidad si bien se relaciona con el tema de la diversidad y la diferencia, algunos han cuestionado esa forma del multiculturalismo como ideología en el capitalismo global, forma vacía que trata a cada cultura local como el colonizador trata al pueblo colonizado. Desde esta perspectiva, el "multiculturalismo" se conforma como un posición logocéntrica y eurocentrista frente a culturales y grupos locales, lo cual lo convierte en forma de racismo velado; se "respeta" al otro mediante el estatuto de "comunidad auténtica" (folklórica), cerrado en sí misma, lo que trae como consecuencia una actitud de distancia y una modalidad de dominación extensa que no interesa en absoluto en conocimiento compartido. La crónica queda como un dispositivo para conocer las diferencias desde sus códigos y sistemas de representación, y para estudiar (con la impronta comunicológica que mencionamos en el punto anterior) las diversas maneras de comprender las interrelaciones culturales en un entorno. $\mathrm{Al}$ evidenciar este sistema múltiple de representación se fortalece el campo de disputa y confrontación por la representaciones y significados; por ello el multiculturalismo (en su sentido más amplio) participa en esa lucha al cuestionar las formas de dominación, fortalece la interpelación al sistema como acto de habla ( $C f$. Dussel, 1993: 13-65).

(d) Un último conjunto de problemas es el que tiene que ver con varias de las críticas a los estudios culturales (y a la crónica como una forma discursiva conveniente útil y funcional para los propósitos que éstos puedan tener). Algunas de éstas, ven ciertas interpretaciones de estos estudios como formas de una "razón indolente" o "perezosa" que ha renunciado al análisis riguroso y ahora deja a la forma 
el peso central, sin importar tanto el contenido como el locus de enunciación. Ello supondría por ejemplo a considerar como legítimamente un texto en este ámbito a quien, con una mera finalidad de difusión. Escuchamos en este sentido el caso de una mujer que habitaba en un chavola, la cual al tener la posibilidad de leer y escribir, pensó en escribir su vida, el cual llegó a ser un éxito e incluso fue aclamado por no pocos culturalistas como narración honesta, auténtica y valiente. En estos, y otros casos lo que se pone a discusión es la frontera entre la sociología, antropología, el periodismo, el testimonio, disputa que por otra parte gusta a no pocos culturalistas ya que por principio siente beneplácito en la ruptura disciplinaria. Con este debate, que ahora solo nombramos se pone de manifiesta el centro de esta disputa: las nuevas forma para definir lo que quiere decir la escritura, los géneros y los dispositivos de enunciación.

Para quienes critican a los estudios culturales, entre otros, Jesús Galindo (2003) en México, Roberto Follari (2003) y Carlos Reynoso (2000) en Argentina, lejos de constituir un proyecto bien articulado capaz de inspirar una nueva forma de ciencia social o de suplantar los saberes dominantes; los EC han acabado por plegarse a las ideas mas convencionales de un postmodernismo desleído, o por cultivar un conformismo sospechosamente unánime ante la cultura de masas. No han aportado todavía los esclarecimientos prometidos, ni han materializado nunca la critica disciplinar de la cual, sin embargo, se jactan.

Uno de los retos de los EC por otra parte parece ser superar la tentación para convertirse en una nueva ortodoxia "débil", "light" que se quede fascinada en sus objetos y que no sepa ser sensible a los nuevos fenómenos culturales que por ejemplo se dan en regiones donde la articulación económica con otras áreas es más fuerte, lo cual llevaré a este campo a nuevas revisiones teóricas. Igualmente aquí la crónica será un instrumento para dar cuenta de esas transformaciones y advertir (como también lo puede hacer) de desplazamientos, fracturas y cartografías. Nos parece que la crónica en tanto dispositivo seguirá siendo un instrumento útil pero que no se puede mimetizar con el estudio de la cultural, en sí mismo es un tipo de discurso (complementario), pero no igual al que puede producir la sociología cultural en su comprensión de modos de producción, sistemas de sentido e identidades. 


\section{Fuentes citadas:}

Aranda Luna, J. (1991) “Entrevista con CM” en Revista Vuelta 174, México. Mayo, pp. $43-46$

Dussel, E. (1993) Apel, Ricoer Rorty y la filosofía de la liberación. México: U de G.

Egan, L. (2001) Carlos Monsiváis: culture and chronicle in contemporary México, Arizona: University of Arizona Press.

Follari, R (2003) Los estudios culturales como teorías débiles. Ponencia presentada en el Congreso LASA, Dallas, Texas. [Ponencia en línea abril 2006]. Disponible en

http://www.portalcomunicacion.com/both/aab/txt/follari 1.pdf

Galindo, J. (2003) Notas para una comunicología posible: elementos para una matriz y un programa de configuración conceptual-teórica, [En línea, febrero 2003]. Disponible en http://www.geocities.com/arewara/arewara.htm

Leñero, V. y Marín C. (1986) Manual de Redacción Periodística. México: Grijalbo.

Mattelar A. y Neveu E. (2004) Introducción a los estudios culturales. Barcelona. Paidos.

Monsiváis, C (1980) A ustedes les consta. Antología de la crónica en México. México: ERA.

Ochoa, A.M. (2002) "Políticas culturales, academia y sociedad" en Mato Daniel (coord.) Estudios y otras prácticas intelectuales latinoamericanas en cultura y poder. Caracas, CLACSO, FACES y Universidad Central de Caracas

Paz, O. (1997) La Llama Doble, Amor y Erotismo, Barcelona, Ed. Seix Barral (Biblioteca de Bolsillo).

Reguillo, R. (2003) "Textos fronterizos. La crónica, una escritura a la intemperie" en revista electrónica DIALOGOS de la Federación Latinoamericana de Facultades de Comunicación Social (FELAFACS) [En línea, noviembre 2003] disponible en www.felafacs.org/dialogos/pdf58/Rossana.pdf

Reynoso C. (2000) Apogeo y decadencia de los estudios culturales. Una visión antropológica. Barcelona: Gedisa.

Rotker, S. (2005) La invención de la crónica. México: FCE / Nuevo Periodismo.

Salazar, J. (2005) “La crónica, estética de una trasgresión” en Razón y Palabra 47. Octubre- Noviembre. México: ITESM-CEM. En línea, disponible en

http://www.razonypalabra.org.mx/anteriores/n47/jsalazar.html

Santos B de S. (2000) A critica da razão indolente... Porto: Afrontamento.

Todorov, T. (1999) La conquista de América. El problema del otro. $10^{\mathrm{a}}$ ed. México: Siglo XXI [1987]

Valenzuela, J,M. (coord.) (2003) Los Estudios Culturales en México. México. CONACULTA/ FCE. (Biblioteca Mexicana) 
Wallerstein, I. (ed.) (1996) Para Abrir las ciencias sociales, México, Siglo XXI. 